\title{
Anisotropic Growth of Porous Anodic Alumina and Its Potential Application in Three-Dimensional Nanostructure Construction
}

\author{
Jinhui Dai, Leiyan Shi, Jintao Tian, Zhibin Zhu, Xiang Huang, Pingwei Wu, and Xiaofei Qu
}

Institute of Materials Science and Engineering, Ocean University of China, Songling Road 238, Shandong Province, Qingdao 266100, China

Correspondence should be addressed to Jinhui Dai, daijh@ouc.edu.cn

Received 15 February 2011; Accepted 22 March 2011

Academic Editor: N. Tabet

Copyright (C) 2011 Jinhui Dai et al. This is an open access article distributed under the Creative Commons Attribution License, which permits unrestricted use, distribution, and reproduction in any medium, provided the original work is properly cited.

The porous anodic alumina was fabricated by means of electrochemical oxidation using $\mathrm{Al}$ foil as a substrate in oxalic acid under constant anodic voltage at room temperature. Prior to anodization, the substrate surface with roughness in microscale was obtained through electrochemical treatment. The tube growth on the substrate was investigated using a scanning electron microscope (SEM). The results showed that the tube growth behavior of the porous anodic alumina was strongly dependent on the surface roughness. In presence of sphere-shaped cavities in microscale (diameter of $5 \sim 30 \mu \mathrm{m}$ ) on the surface of the substrate, the nanotubes were growing essentially away from the center of curvature of each cavity, forming individual growing regions separated by visible interface. The tube branching and tube cutoff were evidently observed in the growing regions and along the interface, respectively. Anisotropic growth of porous anodic alumina suggested its potential application in three-dimensional nanostructure construction.

\section{Introduction}

In recent years, there has been increasing interest in the fabrication of one-dimensional nanostructure materials because of their potential utilization in electronics, optics, and mechanics [1]. Various methods have been developed to fabricate one-dimensional nanostructure materials, such as arc discharge, catalytic CVD growth, and template synthesis, and so forth [2]. In comparison with other porous media such as polymer films, nanochannel glass templates, and micromachined semipermeable membranes, porous anodic alumina is quite attractive [3]. Possessing higher pore density [4], uniform pore size, and high aspect ratio, the wellfabricated porous anodic alumina has been widely used as the template due to the remarkable hardness, thermal and anticorrupted stability together with the potentially low cost and relative simple preparation [5]. In such a case, Al foil with smooth surface after careful polishing was used as a substrate in general and multiple anodizing steps or even mechanical pretexturing $[6,7]$ were performed. The obtained nano tubes were parallel to each other and perpendicular to the smooth surface of the substrate. The ordering degree of the nanopores was found to be scarcely affected by the small surface roughness (nanoscale roughness of $3 \sim 30 \mathrm{~nm}$ ) [8, 9]. In the case of large surface roughness, for instance in micro scale, the nano tube growth behavior was not explored up to now in the literature. It was believed that the surface roughness in micro scale could affect the tube growth to some extent. In this work, nano tube growth behavior of porous anodic alumina fabricated using $\mathrm{Al}$ foil as a substrate with surface roughness in micro scale was investigated. The obtained results suggested a high possibility of preparing three-dimensional nanotemplate and as a result three-dimensional nanostructure.

\section{Experimental Procedure}

Aluminum (Al) foil (purity higher than $99.99 \mathrm{wt} \%$ ) with a thickness of $200 \mu \mathrm{m}$ was used in our experiment. The anodizing sample was cut from the as-received $\mathrm{Al}$ foil to be disc with a diameter of $30 \mathrm{~mm}$. Prior to anodization, the sample was degreased, etched in a $0.24 \mathrm{M} \mathrm{NaOH}$ solution, and rinsed in distilled water. The sample was then electrochemically 


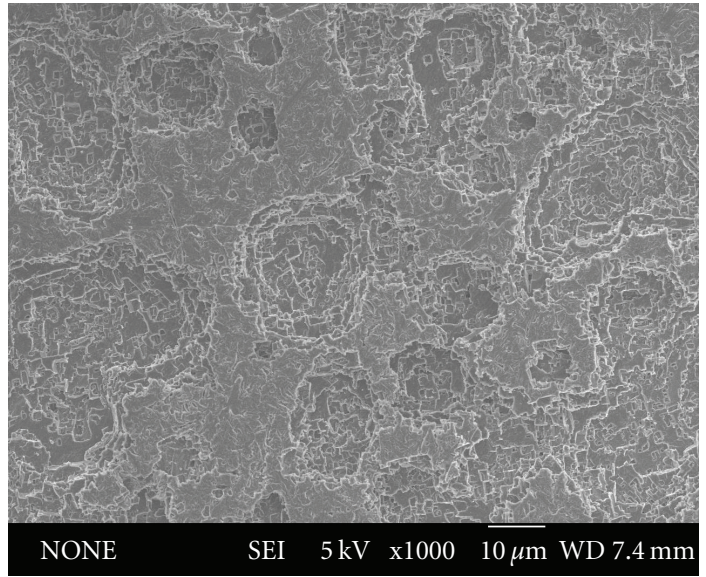

(a)

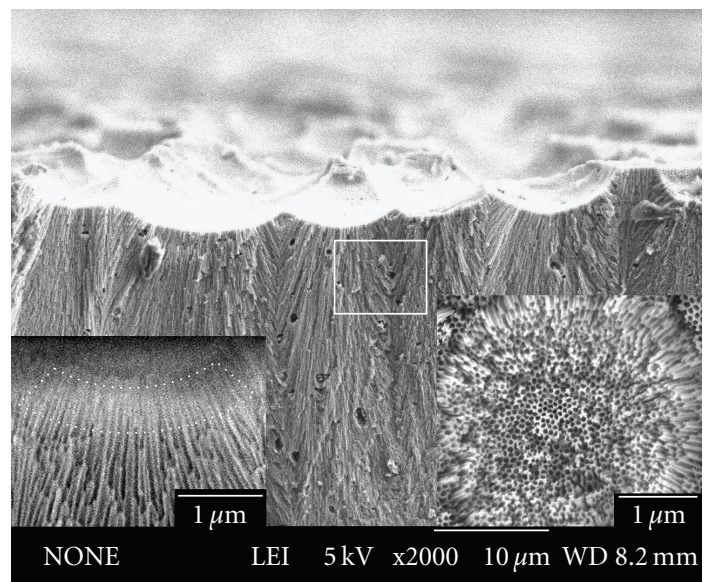

(c)

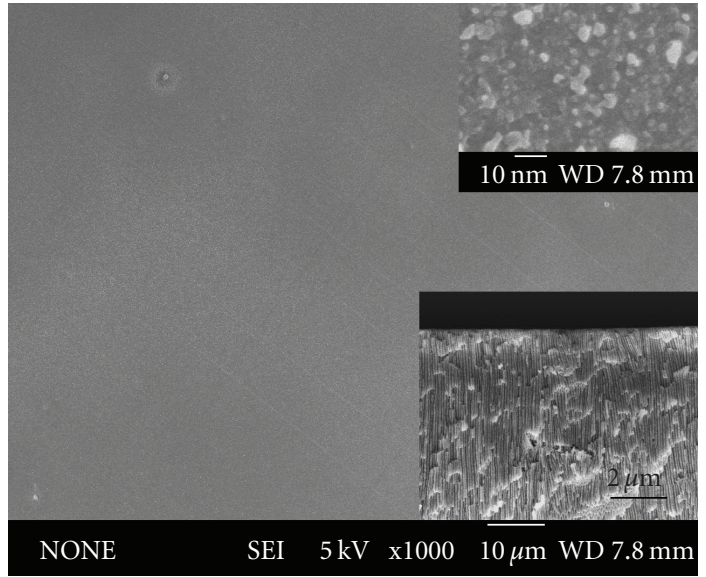

(b)

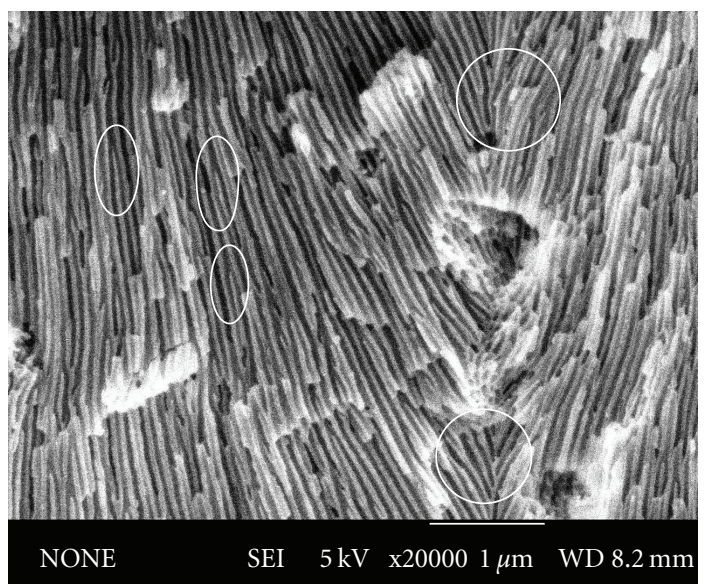

(d)

FIGURE 1: SEM surface morphologies of Al foil as a substrate after electrochemical treatment (a) and chemical polishing (b). SEM crosssection morphologies of the porous anodic alumina prepared by the Al substrate electrochemically treated ((c) and (d)).

treated for $3 \mathrm{~min}$ in a mixture of $\mathrm{HClO}_{4}(70 \sim 72 \mathrm{wt} \%)$ and $\mathrm{C}_{2} \mathrm{H}_{5} \mathrm{OH}\left(\mathrm{HClO}_{4}\right.$ volume fraction of 0.25$)$ to obtain a rough surface. Besides this, the $\mathrm{Al}$ substrate sample with smooth surface was achieved via chemical polishing at $100^{\circ} \mathrm{C}$ for $30 \mathrm{~s}$ in a three-acid solution consisting of $\mathrm{HNO}_{3}(65 \sim 68 \mathrm{wt} \%)$, $\mathrm{H}_{2} \mathrm{SO}_{4}\left(95 \sim 98 \mathrm{wt} \%\right.$ ), and $\mathrm{H}_{3} \mathrm{PO}_{4}$ (larger than $85 \mathrm{wt} \%$ ) in a volume fraction of $10: 15: 75$, and trace $\mathrm{CuSO}_{4} \cdot 5 \mathrm{H}_{2} \mathrm{O}(0.1 \sim$ $0.2 \mathrm{~g}$ per 1 liter three-acid solution).

The anodization was performed in two steps. The sample was first oxidized at a constant voltage of $40 \mathrm{~V}$ in an electrolyte ( $0.3 \mathrm{M}$ oxalic acid solution) at room temperature for $1 \mathrm{~h}$. During this process, the electrolyte was vigorously stirred. The formed alumina layer on the sample surface was then removed in a mixture of phosphoric acid $(5.1 \mathrm{wt} \%)$ and chromic acid $(1.8 \mathrm{wt} \%)$ at $60^{\circ} \mathrm{C}$ for $10 \mathrm{~h}$. After removal, the sample was oxidized again for $4 \mathrm{~h}$ under the same conditions. The remaining $\mathrm{Al}$ in the sample after oxidation was detached using a saturated $\mathrm{CuCl}_{2}$ solution. The obtained porous anodic alumina was then rinsed in distilled water and immersed in a $0.3 \mathrm{M}$ phosphoric acid for about $1 \mathrm{~h}$ at $30^{\circ} \mathrm{C}$ in order to dissolve the bottom barrier-type part of the nanotubes. The microstructure of prepared porous alumina was examined using a scanning electron microscope (SEM, JSM-6700F).

\section{Results and Discussion}

Figures 1(a) and 1(b) show the SEM surface morphologies of the $\mathrm{Al}$ foil as a substrate after surface treatment. As shown in Figure 1(a), large amount of sphere-shaped cavities were distributed disorderly on the sample surface. The diameter of the cavities was estimated to be in the range of $5 \sim$ $30 \mu \mathrm{m}$, a result of surface roughness of the substrate in micro scale due to the electrochemical treatment. The $\mathrm{Al}$ substrate chemically polished displayed a smooth surface, as shown in Figure 1(b). Additional observation of the surface under high magnification revealed roughness in nanoscale (upper right insert of Figure 1(b)).

The SEM cross-section morphologies of the porous anodic alumina prepared by the Al substrate after chemical 


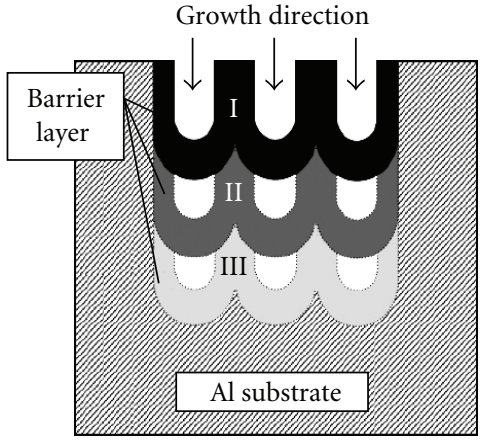

(a)

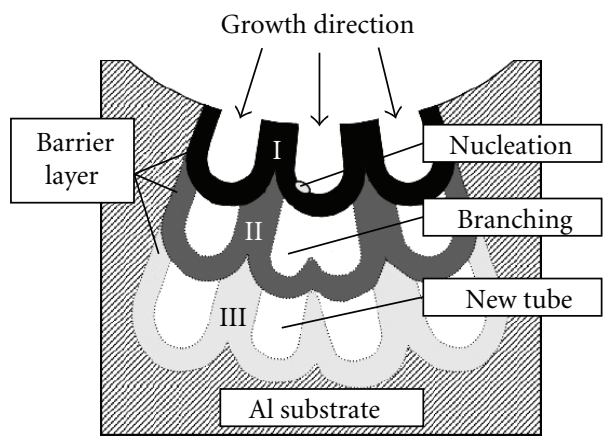

(b)

FIGURE 2: Schematic illustrations of the typical growth process of the nano tubes on smooth surface (a) and rough surface (b), respectively. The diameter of the nano tube was kept to be constant in Figure 2(a) but tends to be larger due to the presence of the micro scale cavity in Figure 2(b).

polishing and two-step anodization were shown in the lower right insert of Figure 1(b). As seen from the insert, the substrate surface was rather smooth. The remaining nanoscale roughness after chemical polishing has been successful removed via the first step anodizing. All the nano tubes were essentially parallel to each other and perpendicular to the substrate surface. Such result was in good agreement with those froms [8], where the pore growth was scarcely affected by the nanoscale surface roughness (less than $30 \mathrm{~nm}$ ) of the substrate. For Al substrate electrochemically treated, the disorderly distributed sphere-shaped cavities on the surface of the substrate after two-step anodization consisted of many nanopores with average diameter of $50 \sim 60 \mathrm{~nm}$ (Figure 1(c)). A typical cavity was shown in lower right insert of Figure 1(c). One interesting thing in the insert was that all the nano pores appeared to be pointing to the center of curvature of the cavity. Such result could be further confirmed via the cross-section view of the sample, as shown in Figure 1(c). The surface of the sample in Figure 1(c) was not smooth but showed some amount of cavities with roughness in micro scale, indicating surface roughness of the Al substrate in such scale being hardly removed via two-step anodization. Besides the surface observation, the cross-section view of the nano tube growth of the sample with rough surface, compared to that of the sample with smooth surface (upper right insert of Figure 1(b)), was quite different. The individual nano tube growth regions separated by visible interface were evidently observed from Figure 1(c), corresponding to the relevant micro scale cavities. The nano tubes in each cavity were not parallel to each other any longer but essentially pointed to the center of curvature of the cavity, in good agreement with the surface observation of the sample (lower right insert of Figure 1(c)). Combing the results from Figures 1(b) and 1(c), we could conclude that the growth direction of the nano tube was definitely perpendicular to the surface of the Al substrate, regardless of the surface roughness either in nanoscale or in micro scale.

Further SEM observation (the rectangle in Figure 1(c)) under high magnification of the nano tube growth was shown in Figure 1(d). All the nano tubes in Figure 1(d) were almost of the same diameter. The tube branching during anodization was evidently observed, as marked by ellipses in this figure. Besides the tube branching, the tube cutoff was noticeably present along the interface of the tube growth regions (see regions marked by circles in this figure). The branching of the nano tubes during anodization has been observed by Sui et al., where Al substrate with smooth surface was used and an anodizing voltage as high as $60 \mathrm{~V}$ was applied [10]. Their results indicated that the creation of branched pores in the sample was a characteristic of the high anodization voltage. This was not in agreement with our results since the anodizing voltage in our case was not as high as $60 \mathrm{~V}$ but only $40 \mathrm{~V}$. The mechanism of the nano tube branching in our experiment could be well understood by considering the micro scale surface roughness of the $\mathrm{Al}$ sample. Figure 2 schematically gives illustrations of the typical growth process of the nano tubes on smooth surface and rough surface, respectively. As seen from Figure 2(a), under constant anodization voltage of $40 \mathrm{~V}$ the nano tube growth on the Al substrate with smooth surface was governed by regular self-organized process, resulting in regular distribution and size uniformity of the formed barrier layer. The diameter of the nano tubes was kept to be constant during the whole growth stage of I to III. For anodization of Al substrate with surface roughness in micro scale (Figure 2(b)) under the same voltage, the formed barrier layer, however, was not regular. The tube diameter was kept not to be constant but tends to be larger due to the presence of larger cavities. In such a case, tube nucleation, as expected, would take place under condition essentially similar to those occurring on the sample surface at the initial anodization stage. With anodization continuing, the nucleated tube was growing to be a branch and finally formed new tube (stage II and III in Figure 2(b)). Thus, it could be concluded that the mechanism of formation of branched nanostructure in Figure 2(b) was not anodizing voltage but the size ununiformity of the barrier layer due to the presence of cavities in micro scale.

The bottom left insert of Figure 1(c) shows a typical SEM morphology of the AAO template fabricated using $\mathrm{Al}$ foil as a substrate with surface roughness in micro scale. While the ordering degree of the nanopores far away from the surface of the AAO template decreased strongly, the nanopores close to the surface of the AAO template was highly ordered 


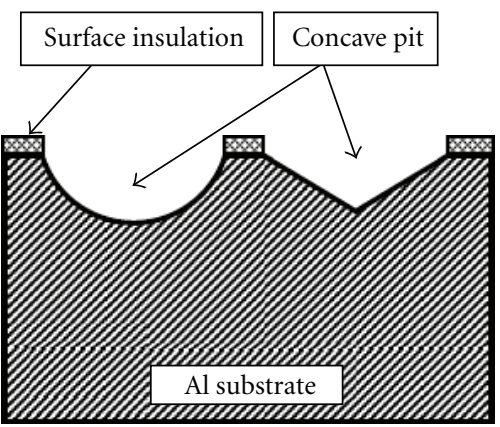

(a)

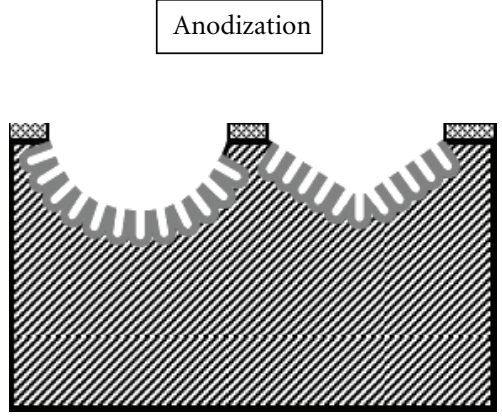

(b)

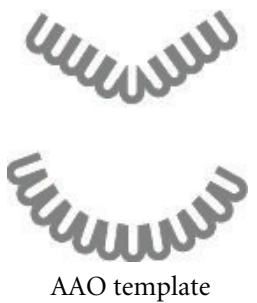

(c)

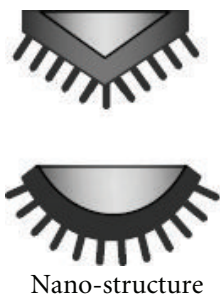

(d)

FIGURE 3: Schematic of design and formation of three-dimensional nanotemplate and nanostructure.

(dash line box in the insert) and radiated from the surface. With these results the preparation of three-dimensional nanotemplate as well as three-dimensional nanostructure was designed as below (Figure 3). With an insulation thin film on the surface of Al foil the concave pits could be formed on the Al substrate surface via erosion method (Figure 3(a)). By applying anodizing treatment for $1-3 \mathrm{~min}$ the threedimensional nanotemplate could be obtained (Figures 3(b) and 3(c)). With such three-dimensional nanotemplate, three-dimensional nanostructures could be achieved via Physical Vapor Deposition, Chemical Vapour Deposition, and Sol-Gel techniques.

\section{Conclusion}

In the present study the porous anodic alumina was fabricated by means of electrochemical oxidation using Al foil as a substrate in oxalic acid under constant anodic voltage at room temperature. Prior to anodization, the substrate surface with roughness in micro scale was obtained through electrochemical treatment. The SEM results indicated that the tube growth behavior was strongly dependent on the surface roughness. For Al substrate with surface roughness in nanoscale, the tube growth was scarcely affected by the surface roughness, in good agreement with the results in the literature. In presence of sphere-shaped cavities in micro scale (diameter of $5 \sim 30 \mu \mathrm{m}$ ) on the surface of the substrate, the individual tube growing regions separated by visible interface was formed. The tube growth direction was found to be definitely perpendicular to the surface of the Al substrate, regardless of the surface roughness either in nanoscale or in micro scale. The tube branching was evidently observed in the growing regions. The mechanism of formation of such branched nanostructure was schematically illustrated to be related to the size ununiformity of the barrier layer due to the presence of cavities in micro scale. Meanwhile, these results suggested a high possibility of preparing threedimensional nanotemplate and as a result three-dimensional nanostructure.

\section{Acknowledgment}

This work was funded by the Shandong Province Key Science and Technique Program of China (Grant no.
2009GG10003039) and Qingdao Science and Technique Program of China (10-3-4-1-9-jch).

\section{References}

[1] H. Masuda and K. Fukuda, "Ordered metal nanohole arrays made by a two-step replication of honeycomb structures of anodic alumina," Science, vol. 268, no. 5216, pp. 1466-1468, 1995.

[2] X. Liu, J. Wang, J. Zhang, and S. Yang, "Fabrication and characterization of $\mathrm{Zr}$ and $\mathrm{Co}$ co-doped $\mathrm{LiMn}_{2} \mathrm{O}_{4}$ nanowires using sol-gel-AAO template process," Journal of Materials Science: Materials in Electronics, vol. 17, no. 11, pp. 865-870, 2006.

[3] D. Gong, V. Yadavalli, M. Paulose, M. Pishko, and C. A. Grimes, "Controlled molecular release using nanoporous alumina capsules," Biomedical Microdevices, vol. 5, no. 1, pp. 75-80, 2003.

[4] W. Chen, J. S. Wu, J. H. Yuan, X. H. Xia, and X. H. Lin, "An environment-friendly electrochemical detachment method for porous anodic alumina," Journal of Electroanalytical Chemistry, vol. 600, no. 2, pp. 257-264, 2007.

[5] X. J. Xu, G. T. Fei, L. Q. Zhu, and X. W. Wang, "A facile approach to the formation of the alumina nanostructures from anodic alumina membranes," Materials Letters, vol. 60, no. 19, pp. 2331-2334, 2006.

[6] H. Asoh, K. Nishio, M. Nakao, T. Tamamura, and H. Masuda, "Conditions for fabrication of ideally ordered anodic porous alumina using pretextured Al," Journal of the Electrochemical Society, vol. 148, no. 4, pp. B152-B156, 2001.

[7] M. T. Wu, I. C. Leu, and M. H. Hon, "Effect of polishing pretreatment on the fabrication of ordered nanopore arrays on aluminum foils by anodization," Journal of Vacuum Science and Technology B, vol. 20, no. 3, pp. 776-782, 2002.

[8] C. U. Yu, C. C. Hu, A. Bai, and Y. F. Yang, "Pore-size dependence of AAO films on surface roughness of Al-1050 sheets controlled by electropolishing coupled with fractional factorial design," Surface and Coatings Technology, vol. 201, no. 16-17, pp. 7259-7265, 2007.

[9] T. Kikuchi, M. Sakairi, and H. Takahashi, "Growth of porous type anodic oxide films at micro-areas on aluminum exposed by laser irradiation," Electrochimica Acta, vol. 54, no. 27, pp. 7018-7024, 2009.

[10] Y. C. Sui, J. A. González-León, A. Bermúdez, and J. M. Saniger, "Synthesis of multi branched carbon nanotubes in porous anodic aluminum oxide template," Carbon, vol. 39, no. 11, pp. 1709-1715, 2001. 

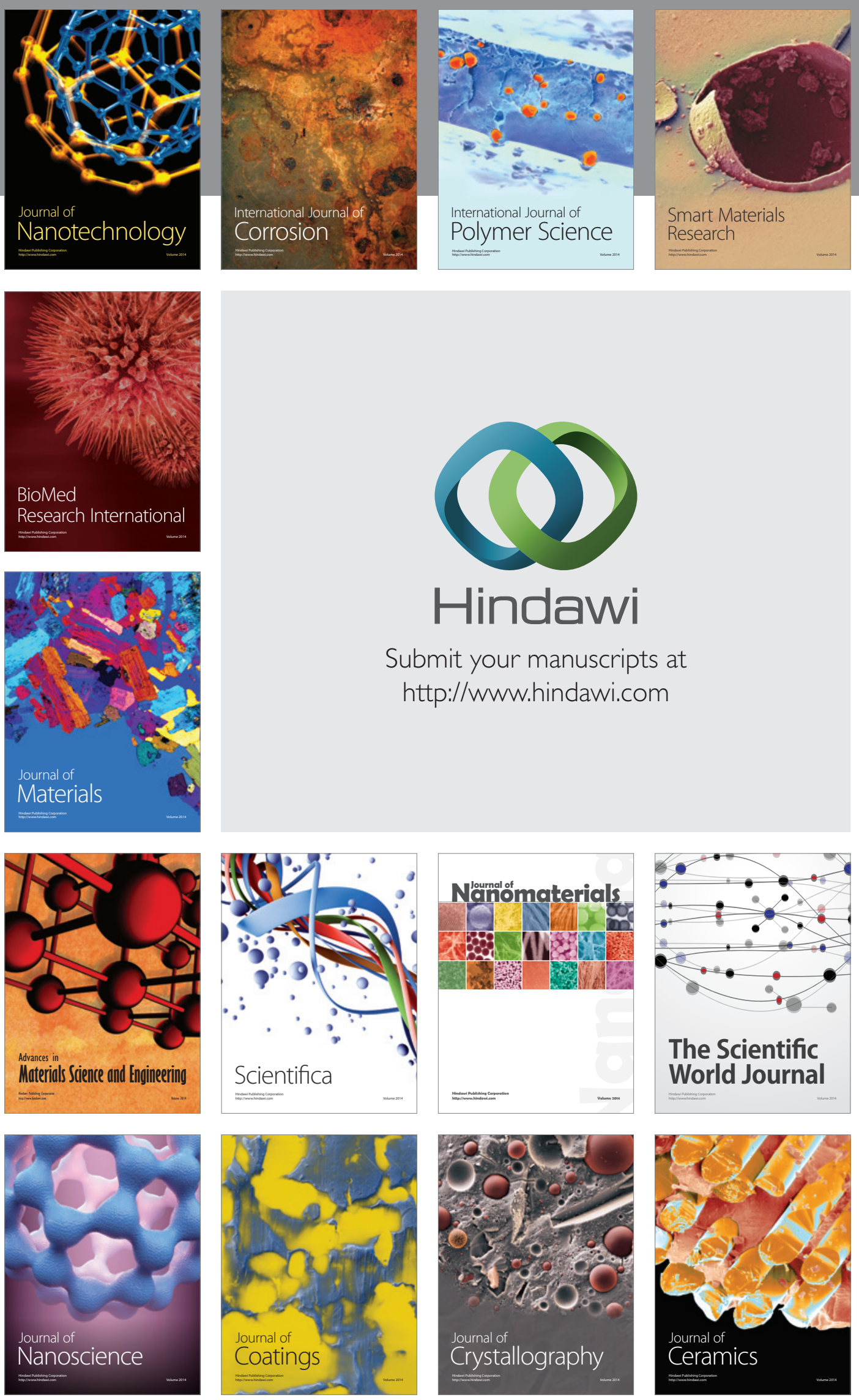

The Scientific World Journal

Submit your manuscripts at

http://www.hindawi.com

\section{World Journal}

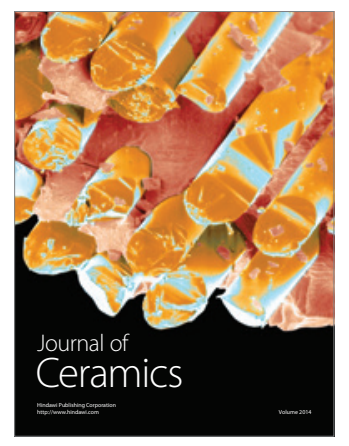

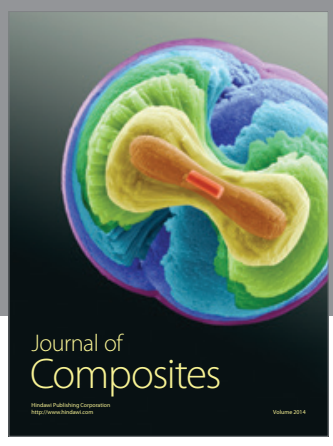
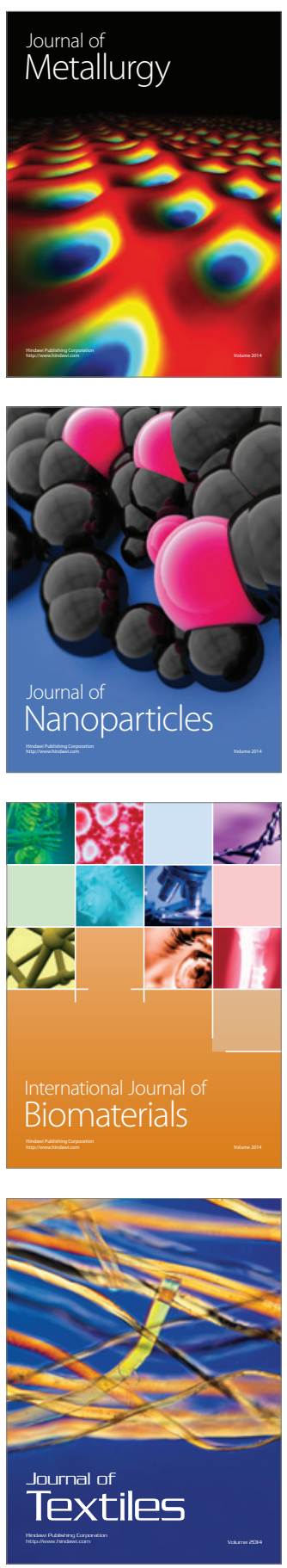